\title{
Adenovirus redirected
}

\author{
Savio L.C. Woo
}

Although adenovirus can transduce a wide spectrum of mammalian cells, it is particularly suited as a transfer vehicle for gene delivery into epithelial cell types via viral receptors preferentially expressed on the surface of those cells. The ability to alter this natural tropism permits adenovirusmediated gene transfer into alternative cell types, which will greatly broaden the scope of target diseases that can be treated by such vectors. In this issue, two papers ${ }^{1,2}$ report for the first time the successful alteration of the adenoviral tropism by modifying the viral epitope for cellular receptor binding.

The two papers describe similar strategies to achieve opposite objectives. It is well established that the binding of adenovirus to its yet-to-be-identified cellular receptor is mediated through the knob domain of the fiber capsid protein. Wickham et al.' (pp. $1570-1573$ ) have succeeded in greatly broadening the host cell range of recombinant adenoviruses by inserting a polycationencoding region into the carboxy-terminal end of the fiber gene. As most mammalian cell types express heparin/heparan sulfate containing cellular receptors, the genetically modified adenovirus is capable of transducing a wide variety of cell types in culture with efficiencies similar to epithelial cells. In a contrasting approach, Douglas et al. ${ }^{2}$ (pp. 1574-1578) chemically conjugated the Fab fragment of a neutralizing anti-knob monoclonal antibody with folate. They then used

Savio L.C. Woo is professor and director at the Institute for Gene Therapy and Molecular Medicine, Mount Sinai School of Medicine, New York, NY10029 (swoo@smtplink.mssm.edu). the molecular conjugate to block virus binding with its natural cellular receptor, while at the same time endowing the virus with a novel epitope for binding to the folate receptor. As folate receptor expression is elevated in a number of tumor cell types, they were able to demonstrate that tumor cells that are refractory to adenovirus infection could be successfully transduced. Thus, without genetically altering the viral genome, they redirected the viral tropism to completely different cell types.

While both reports are ground-breaking studies, there are critical issues that need to be resolved before the potential for such technologies to treat various diseases can be realized. Although Wickham et al. clearly demonstrated effective transduction of alternative cell types in vitro with the polycationmodified vector, they did not present in vivo gene transfer data; thus, it is not yet clear whether such vectors will be useful for improving the efficiency of therapeutic gene delivery to solid tissues and organs. One limitation for systemic delivery might be that the polycations on the genetically modified virus would be saturated with polyanions in the blood, thereby "neutralizing" the capacity of the virus for subsequent target cell transduction. From a technical standpoint, problems resulting from the "stickiness" of these viruses, such as virus production, reduced viral titer, and subsequent handling, must also be addressed.

Stickiness is certainly not an issue for the modified adenovirus reported by Douglas et al. By a clever simple step, they were able to redirect the virus to a completely different cellular receptor. To achieve a broader host cell range, it is theoretically possible to substitute folate for a variety of ligands for various cellular receptors. Thus, their strategy is very attractive as it offers a means of broadening the host cell range of the virus without sacrificing target cell specificity. The critical issues that need to be addressed here are the effect of such manipulations on the viral titer, and a clear demonstration that such redirecting of viral tropism can actually be achieved in vivo with adequate efficiency and specificity.

Theoretical and technical limitations notwithstanding, both papers represent significant conceptual advancements that are critically needed in the field of gene therapy. Together with recent progress made in recombinant adenoviral vector development by further deletion of the $\mathrm{E} 4$ region $^{3,4}$ and all of the viral genes ${ }^{5}$, as well as temporary host immunomodulation ${ }^{6,7}$ to better achieve persistence of virally transduced cells in vivo, the recombinant adenovirus is becoming a much improved vector. Emphasizing such scientific and technological advancements before their clinical application is certainly within the spirit of the report by the Motulsky-Orkin Committee on human gene therapy.

1. Wickham, T.J., Roelvink, P.W., Brough, D.E., and Kovesdi, I. et al. 1996. Nature Biotechnology 14:1570-1573

2. Douglas J.T., et al. 1996. Nature Biotechnology 14:1574-1578.

3. Krougliak, V. and Graham, F.L. 1995. Hum. Gen. Ther. 6:1575-1586.

4. Wang et al. 1995. Gene Ther. 2:775-783.

5. Mitani, K., Graham, F.L., Caskey, C.T., and Kochanek, S. 1995. Proc. Natl. Acad. Sci. USA. 92:3854-3858.

6. Fang et al. 1995. Hum. Gene Ther 6:1039-1044.

7. Yang, Y., Trinchieri, G., and Wilson, J.M. 1995. Nature Med. 1:890-893.

8. Orkin, S.H. and Motulsky, A.G. 1995. Report and Recommendations of the Panel to Assess the NIH Investment in Research on Gene Therapy. NHH, Bethesda, MD.

\section{The 2,5'A antiviral system in plants: A dose of mammalian medicine}

\section{Roger N. Beachy}

Plant viruses can cause yield losses as high as $90 \%$ in selected crops during epidemics and can be particularly devastating to agriculture in tropical countries; yield losses of 1-10\%

Roger N. Beachy is head of the Division of Plant Biology, The Scripps Research Institute, 1066 North Torrey Pines Road, La Jolla, CA 92037 (beachy@scripps.edu). are also not uncommon in temperate parts of the world. The development of transgenic plants containing components of the interferon-induced antiviral system of mammalian cells is the latest in a long line of strategies for creating plants resistant to a broad range of viruses. In this issue, Ogawa et al' report the development of transgenic tobacco plants that produce human-derived 2',5'-oligoadenylate synthetase (2-5Aase) and ribonuclease $L, a$ single-stranded RNAse that is activated by 25Aase: Both of these enzymes are components of an interferon-induced antiviral system that protects mammalian cells from infection by certain viruses.

A second group, under the direction of Robert Silverman at the Cleveland Clinic Research Institute (Cleveland, $\mathrm{OH}$ ), has independently carried out analogous studies with similar results. Their data were recently 